die Hälfte $(575,39)$ von jener, welche ich für das Aequivalent des Wolframs gefunden habe.

Und so erlaube ich mir denu schliesslich für das Aequivalent des Wolframs die Zahl 1150,78 vorzuschlagen. Danach wird die procentische Zusammensetzung der Wolframsiaure:

$$
\begin{aligned}
& \mathrm{W}=79,321 \\
& 0=20,679
\end{aligned}
$$

und die des Wolframoxydes $\left(\mathrm{WO}_{2}\right)$ :

$$
\begin{aligned}
& W=85,19 \% \\
& 0=14,807 .
\end{aligned}
$$

\title{
XXVII.
}

\section{Untersuchungen über die Zusammensetzung der Tantalerze.}

\author{
Von
}

\section{R. Hermann.}

1) Ueber die Zusammensetzung des Tantalits und Columbits, so wie übtr eine Methode die verschiedenen Oxyde des Eisens und Cers in ihren in Säuren unlöslichen Verbinlungen mit tantalälnlichen Substansen $\approx u$ bestimmen.

Bisher uahm man an, dass der Tantalit dieselbe stöchiometrische Constitution besitze, als der Columbit und dass diese Mineralien nach den Formeln:

zusammengesetzt wären.

$$
\begin{aligned}
& \dot{\mathrm{R}}_{2} \ddot{\mathrm{T}}_{\mathrm{a}_{3}} \text { und } \\
& \dot{\mathrm{R}}_{2}\left\{\begin{array}{l}
\ddot{\mathrm{N}}_{\mathrm{b}_{3}} \\
\ddot{\mathrm{P}}_{\mathrm{p}_{3}}
\end{array}\right.
\end{aligned}
$$

Nit dieser Annahme stimmt aber der Imstand nicht wohl überein, dass Tantalit und Columbit wesentlich verschiedene Krystallformen besitzen.

Nach Hern v. Nordenskiöld haben die Tantalite von Kimito und Tamela folgende Formen (Tantalit von Kimito):

sirystallsystem ein- und einaxig.

Figur 1.

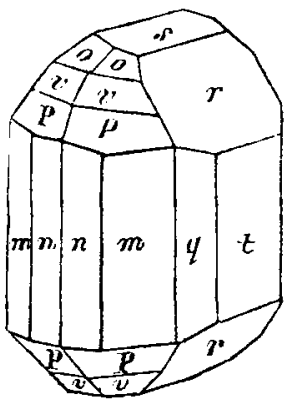


Oktaëder.

$$
\begin{aligned}
& P=(a: b: c)=126^{\circ} 0^{\prime}, 112^{\circ} 30^{\prime}, 91^{\circ} 42^{\prime}, \\
& v=\left(a: b: \frac{2}{3} c\right)=134^{\circ} 38^{\prime}, 89^{\circ} 52^{\prime}, 107^{\circ} 27^{\prime} \\
& o=\left(a: b: \frac{1}{3} c\right)=141^{\circ} 48^{\prime}, 73^{\circ} 37^{\prime}, 119^{\circ} 4 \tilde{b}^{\prime}
\end{aligned}
$$

Vertikale Prismen.

$$
\begin{aligned}
& q=\left(a: \frac{1}{3} b: \infty c\right)=54^{\circ} 10^{\prime} \\
& m=(a: b: \infty c)=113^{\circ} 48^{\prime} \\
& n=(a: 6 b: \infty c)=122^{\circ} 54^{\prime}
\end{aligned}
$$

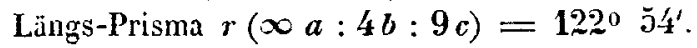

Basis $\boldsymbol{s}$.

Lüngsfläche $t$.

Bei den Columbiten dagegen sind folgende Formen beob)-achtet worden (Columbit rom Ilmengebirge):

Figar 2.

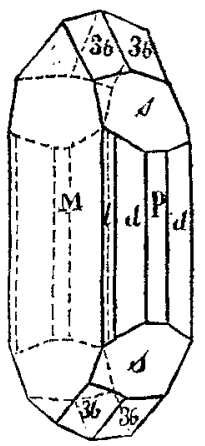

Figur 3.

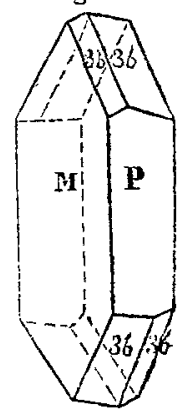

Krystallsystem ein- und einaxig.

Oktaëder.

$$
\begin{aligned}
\frac{1}{3} b & =\left(a: \frac{1}{3} b: c\right)=62^{\circ} 16^{\prime}, 160^{\circ} 29^{\prime}, 121^{\circ} 38^{\prime}, \\
a & =(a: b: c)=117^{\circ} 58^{\prime}, 102^{\circ} 58^{\prime}, 107^{\circ} 56^{\prime}, \\
2 b & =(a: 2 b: c)=126^{\circ} 42^{\prime}, 80^{\circ} 24^{\prime}, 124^{\circ} 41^{\prime}, \\
3 b & =(a: 3 b: c)=150^{\circ} 17^{\prime}, 86^{\circ} 52^{\prime}, 100^{\prime} 49^{\prime} .
\end{aligned}
$$

Vertikale Prismen.

$$
\begin{aligned}
& d=\left(a: \frac{1}{3} b: \infty c\right)=43^{\circ} 48^{\prime}, \\
& f=(a: b: \infty c)=100^{\circ} 16^{\prime}-100^{\circ} 40^{\prime} \\
& g=\left(a: 1 \frac{2}{3} b \infty c\right)=126^{\circ} 46^{\prime} \\
& h=(a: 1+\infty: \infty c)=130^{\circ} 32^{\prime}, \\
& i=(a: 2 b: \infty c)=134^{\circ} 58^{\prime} \\
& k=(a: 3 b: \infty c)=151^{\circ} 46^{\prime}
\end{aligned}
$$




$$
\begin{aligned}
& \text { Quer-Prismen. } \\
& m=\left(a: \infty b: 1 \frac{1}{3} c\right)=70^{\circ} 50^{\prime} \text {, } \\
& n=\left(a: \infty b: \frac{2}{3} c\right)=109^{\circ} 35^{\prime} \text {, } \\
& p=\left(a: \infty b: \frac{1}{3} c\right)=141^{\circ} 8^{\prime} \text {, } \\
& r=\left(a: \infty b: \frac{1}{6} c\right)=160^{\circ} \text {. } \\
& \text { Längs - Prismen. } \\
& s=\left(\infty a: \frac{1}{2} b: c\right)=59^{\circ} 20^{\prime} \text {, } \\
& t=\left(\infty a: \frac{3}{4} b: c\right)=80^{\circ} \text {, } \\
& u=(\infty a: b: c)=97^{\circ} 27^{\prime}-98^{\circ} 14^{\prime} \text {, } \\
& v=\left(\infty a: 1 \frac{2}{3} b: c\right)=139^{\circ} 6^{\prime} \text {. } \\
& \text { Querlläche } \boldsymbol{M}=(a: \infty \text { b: } \infty c) \text {. } \\
& \text { Lāngsflüche } P=(\infty a: b: \infty c) \text {. }
\end{aligned}
$$

Diese Formen des Tantalits und Columbits lassen sich nicht in Uebercinstimmung bringen. Man müsste daher annehmen, entweder, dass Tantalsāure und Niob-, Pelop- und llmen-Säture dimorph wären, oder dass Tantalit und Columbit wesentlich verschiedene chemische Constitution besitzen. Mir schien letztere Ansicht die wahrscheinlichere zu sein. Es war daher zu prüfen, ob im Tantalite oder im Columbite, neben den $0 x y-$ dulen von Eisen und Mangan, nicht vielleicht auch die Oxyde dieser Basen vorhanden wären.

Bei der Beantwortung dieser Frage stiess man sogleich auf das Hinderniss, dass bisher noch keine Methode bekannt war, um die verschiedenen Oxyde der genannten Metalle in ihren Verbindungen mit tantalälınlichen Substanzen zu bestimmen. Jedoch hatle ich bei meinen Untersuchungen der Turmaline und Epidote bereits Gelegenheit solche Bestimmungen bei in Säuren unlöslichen Silicaten auszuführen. Das Verfahren, welches damals zu ganz brauchbaren Resultaten führte, scheint auch bei den Tantalaten anwendbar zu sein. Es bestand nämlich darin, die feingeriehenen Hineralien mit ibrer $\mathbf{5}$ fachen Menge wasserfreien Boraxes zusammen zu schmelzen, das so erzengte klare Glas unter Alsschluss von Luft in Salzsäure zu lösen, die saure salzsaure Lösung mit Kupfer zu kochen und aus der Menge des dabei gelösten Kupfers die Menge der in der Lösung enthaltenen 0xyde vou Eisen, Mangan, Cer oder Uran zu berechnen. 
Vor Anwendung dieser Methode waren aber erst folgende Fragen zu beantworten:

1) Können Tantalate mit Borax zusammengeschmolzen werden, ohne dass dabei eine Aenderung des ursprünylichen Oxydation;-Zustandes der in ihnen enthaltenon metallischen Basen eintritt?

2) Wie verbalten sich tantalähnliche Säuren und die sie so häufig begleitende Titansäure in Berübrung mit Salzsäure und Kupfer?

Was die erste Frage anbelangt, so war nicht einzusehen, weshalb sich die Tantalate der verschiedenen Oxyde des Eisens u. s. w. beim Zusammenschmelzen mit Borax anders verhalten sollten als die Silicate dieser Basen. Bei letzteren habe ich aber schon nachgewiesen, dass ihr Gemenge mit wasserfreiem Borax, wenn man es in doppelt verschlossenen Platintiegeln in Mulfelofen schmilzt, keine Gewichts - Veränderung erleidet, vorausgesetzt, dass in den Mineralien keine füchtigen Bestandtheile vorhanden waren. Es kounte dabei also auch keine Oxydation oder Reduktion der in diesen Mineralien enthaltenen metallischen Basen stattgefunden baben, weil in diesen Fälen merkliche Gewichts-Veränderungen eintreten müssten. Uebrigens lässt sich leicht eine Vorrichtung ausdenken, welche einen Luftwechsel in dem Tiegel, in welchem die Schmelzung mit Borax vorgenommen wird, vollständig verhindert.

Zu diesem Versuch nehme ich gewühnlich 20 Gran Mineral und 100 Gran wasserfreien Borax. Das zusammengeschmolzene Glas muss ganz lilar sein und keine $S_{p}$ ur ungelösten Minerals mehr enthalten. In letzterem Falle muss es unter Zusatz von noch mehr Borax umgeschmolzen werden.

Das klare Glas wird fein zerrieben. Hieranf füllt man einen kleinen Glaskolben, der 4 Unzen Wasser fassen kann, zur Hälfte mil einem Gemische ans 1 Theil concentrirter Salzsäure und 2 Theilen Wasser, wirn in dieses Gemisch einige Stückchen reinen Kalkspaths und verschliesst die Oeffnung des Kolbens locker mit einem Pfropfen. Die entweichende Koblensäure treibt alle atmosphärische Luft aus dem Kolben. Noch ehe der Kalkspath ganz gelöst ist, setze man das Glaspulver zur Säure und schüttele gut durcheinander. Hierauf fülle man den Kolben bis in seinen engen Hals mit demselben Sämre- 
Gemisch an, setze zur Flüssigkeit einige Streifen ganz reinen zuvor genau abgewogenen Kupferbleches, verschliesse den Kolben mit einem gebogenen Glasrohr, dessen offene Mündung unter Wasser geleitet wird, erhitze die Flüssigkeit langsam bis zum Kochen und lasse das Kochen 5 Minuten lang anhalten. Jetzt giesse man den Inhalt des Kolbens in eine Schale aus, nehme das Kupferblech sogleich aus der Flüssigkeit, spüle es gut ab, trockne und wiege es.

Hieniluei wird angenommen, dass Kupfer von kochender Salzsäure unter Abschluss von Luft nicht angegriffen wird; dass dagregen Auflïsung ron Kupfer erfolgt, wenu die Flüssiglicit Chloride von Eisen, Mangan, Cer oder Uran enthält. Hierbei werden die Chloricle zu Chlorüren und ron jedem Atom freiwerdenden Chlors wird 1 Atom Küpfer gelüst. Es entspricht also jedes Atom gelösten Kuprers je einem Atome der in dem untersuchten Minerale enthalten gewesenen Oxyde von Eisen, Hangan, Cer oder Uran.

Ausser den genanuten 0xyden befinden sich aber in den hier in Betracht hommenden Nineralien auch noch. Tantal-, Pelop-, Niob-, Jimen- und Titansüure. Da das Verhalten dieser Substanzen in Berührung mit Kupfer und Salzsäure bisher noch nicht untersucht war, so musste zuvor ausgemittelt werden, ob sie sich dabei nicht vielleicht eben so verhielten als in $\mathrm{Be}-$ rührung mit Salzsäure und Zink. Auch war zu untersuchen, ol) nicht vielleicht Kupfer beim Kochen blos mit Borax und Salzsäıre, doch in geringer Menge gelöst werde. Jch habe daher folgende Versuche angestellt:

1) 100 Gran Boraxglas wurden in 4 Unzen verdümnter Salzsäure gelüst, unter Abschluss der Luft mit 50 Gran Kupferblech erhitzt und 5 Yinuten lang geliocht. Das Kiupfer verlor dabei am Gewicht nur 0,05 Gran.

2) 20 Gran Tantalsäure wurden mit $\mathbf{1 0 0}$ Gran wasserfreien Baryts zusammengeschmolzen, das klare Glas gepulvert und wie vorstehend angegeben behandelt. Beim Lusen des Glases in Salzsäure schied sich der grōsste Theil der Tantalsäure in weissen Flocken ab. Beim Kochen mit Kupfer veränderte die Tantalsäure ilsre Farbe nicht. Das hupfer batte am Gewicht nicht mehr verloren als bei dem Versuche No. 1. Die Tantalsäurc wird also beim Kochen mit Salzsäure und Kupfer nicht verändert. 
3) Pelop - und Ilmensïure verhielten sich beim Kochen mit Salzsiure und Kupfer ganz so wie die Tantalsäure.

4) Niobsäure aus Aeschynit gab mit Borax ein Glas, welches sich vollständig in Salzsảure löste. Auch beim Kochen dieser Lüsung schied sich keine Niobsäure aus. Das Kupfer wurde dabei etwas angegriffen und verlor bei der Anwendung ron 20 Gran Niobsäure 0,20 Gran an Gervicht. Ammoniak schlug die gelöste Niobsäure mit rosenroher Farbe nieder. Beim Stehen an der Luft wurde dieses Hydrat wieder wciss. Bei näherer Untersuchung ergab es sich abcr, dass die angewandte Niobsäure etwas Titansänre enthielt, wodurch die Einwirkung auf das Kupfer veranlasst worden war. Reine Niobsäure wird bei Eintvirkung von Kupfer und Salzsäure nicht verändert.

5) 20 Gran Titansüure wurde mit 100 Gran trocknem Borax zusammengesclumolzen. Es entstand ein klares gelles Glas, welches sich vollstindig in verdünnter Salzsăure aufloste. Durch Einwirkung des Liupfers nahm die Lösung eine rosenrothe Färbung an.

Dabei wurde das Kupfer stark angegriffen. Nach 5 Minuten lang fortgesetztem kochen, hatte das Kupfer 2,20 Gran an Gewicht verloren. Die salzsaure Lösung gab mit Ammoniak einen purpurrothen Niederschlag eines Gemenges aus Titansäurehydrat und titansaurem Titanoxyd.

Dieses Verhalten der Titansäure gegen Kupfer, darf bei den Versuchen über die 0xydations-Zustände der metallischen Basen der Tantalerze nicht überselien werden, da diese Mineralien häufig Titansäure enthalten. In diesen Fällen muss man stets besondere Gegenversuche anstellen, um die Quantität des Kupfers $z u$ bestimmen, welche durch dic Einwirkung der Titansäure gelüst wurde.

Als Resultat meiner Versuche über die Quantitäten von lípfer, welche beim kochen mit Lösungen verschiedener Tanta!Erze in Salzsäure gelüst wurden, erhielt ich folgende Zahlen:

Angewandtes Mineral 20 Gran. Borax, wasserfrei, 100 Gran. Liupfer 50 Gran.

1) Columbil vom Ilmen-Gebirge.

Gelüstes Kupfer 0,20 Gran

Für 0xydation

Rest $\frac{, 05, ",}{0,15}$ 
2) Columbit von Midlletown in Nordamerika.

Gelöstes Kupfer 0,40 Gran

Für 0xydation $\frac{0,0 \breve{5}}{\text { Rest }}$,",

100 Gran Mineral würden also 1,75 Gran hupfer gelóst, mithin 4,42 Gran Eisenoxyd enthalten haben.

3) Tantalit von Kimito in Finnland.

$\begin{array}{ccc}\text { Gelöstes Kupfer } & 0,75 \text { Gran } \\ \text { Für Oxydation } & \begin{array}{r}0,05 \\ \text { Rest }\end{array} & 0,70\end{array}$

100 Gran Mineral würden also 3,50 Gran hupfer gelöst, mithin 8,84 Gran Eisenoxyd enthalten haben.

\section{4) Asrhynit vom llmengebirge. \\ Gelöstes Kupfer 1,83 Gran \\ Fïr 0xydation 0,05 , \\ Für Titansïure 0,57, \\ Rest $\frac{0,21}{1,21} "$}

100 Gran Aeschynit würden also 6,05 Gran Kupfer gelōst, mithin 22,20 Gran Ceroxyd enthalten haben.

Ein Blick auf diese Resultate lehrt, dass die metallischen Basen sich im Columbite im Zustande von Oxydul befinden. Denn, obgleich die Quantităt des Eisenoxydes im amerikanischen Columbit bis auf 4,42 p. C. steigt, so füllt es doch im ilmenschen Columbit bis auf 1.89 p. C. Der Gehalt des Columbits an Eisenoxyd ist daher nur zufällig.

Dasselbe kann man aber nicht von dem Gehalte des Tantalits an Eisenoxyd sagen. Dieses steigt bis über 8 p. C. und beträgt mehr als die Hälfte aller im Tantalite enthaltenen Basen. Man muss daher den Tantalit als ein Doppelsalz betrachten, in dem sowohl einatomige als dreiatomige Basen enthalten sind. Der Tantalit hätte demnach eine ganz verschiedene chemische Constitution wie der Columbit und dieser Umstand erklürt dann auch ganz einfach die verschiedene Form beider Mineralien.

Der Tantalit von Kimito ist bereits früher von Berzelius untersucht worden. Er bestand aus :

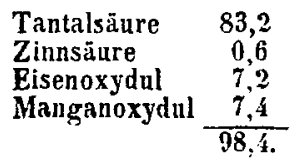


100 Theile Nineral würden also 0,75 Gran Kupfer gelöst, daher 1,89 Gran Eisenoxyd enthalten haben.

Da aber in diesem Mineral das Aequivalent von $8,84 \mathrm{p}$. C. Eisenoxyd an dreiatomigen Basen enthalten ist, so gestaltet sich diese Analyse wie folgt:

\begin{tabular}{|c|c|c|c|c|c|}
\hline \multirow{4}{*}{$\begin{array}{l}\text { Tantalsäure } \\
\text { Zinnsiurure } \\
\text { Eisenoxyd } \\
\text { Manganoxyd } \\
\text { Manganoxydul }\end{array}$} & \multicolumn{3}{|c|}{ Sauerstoff. } & \multicolumn{2}{|c|}{$\begin{array}{l}\text { Gefundene Angenommene } \\
\text { Proportion. Proportion. }\end{array}$} \\
\hline & $\begin{array}{r}83,20 \\
0,60\end{array}$ & $\left.\begin{array}{r}10,86 \\
0,12\end{array}\right\}$ & 10,98 & 12,52 & 12 \\
\hline & 8,00 & & 2,63 & 3,0 & 3 \\
\hline & $\begin{array}{l}0,79 \\
6,69\end{array}$ & $\begin{array}{l}0,23\} \\
1,50\end{array}$ & & & 2 \\
\hline & $99,28$. & & & & \\
\hline
\end{tabular}

Der Tantalit wäre demnach nach der Formel:

zusammengesetzt.

$$
\dot{\mathrm{R}}_{2} \ddot{\mathrm{T}} \mathrm{a}_{3}+\dddot{\mathbb{R}} \ddot{\mathrm{T}} \mathrm{a}_{3}
$$

Dagegen bleibt die Zusammensetzung der Columbite ganz unverändert so, wie ich bereits in diesen Journale Bd. XLIII, S. 208 angegeben habe. Danach enthielten sie folgende Bestandtheile:

\section{1) Columbit von Middletown in Nordamerika.}

Spec. Gew. 5,80.

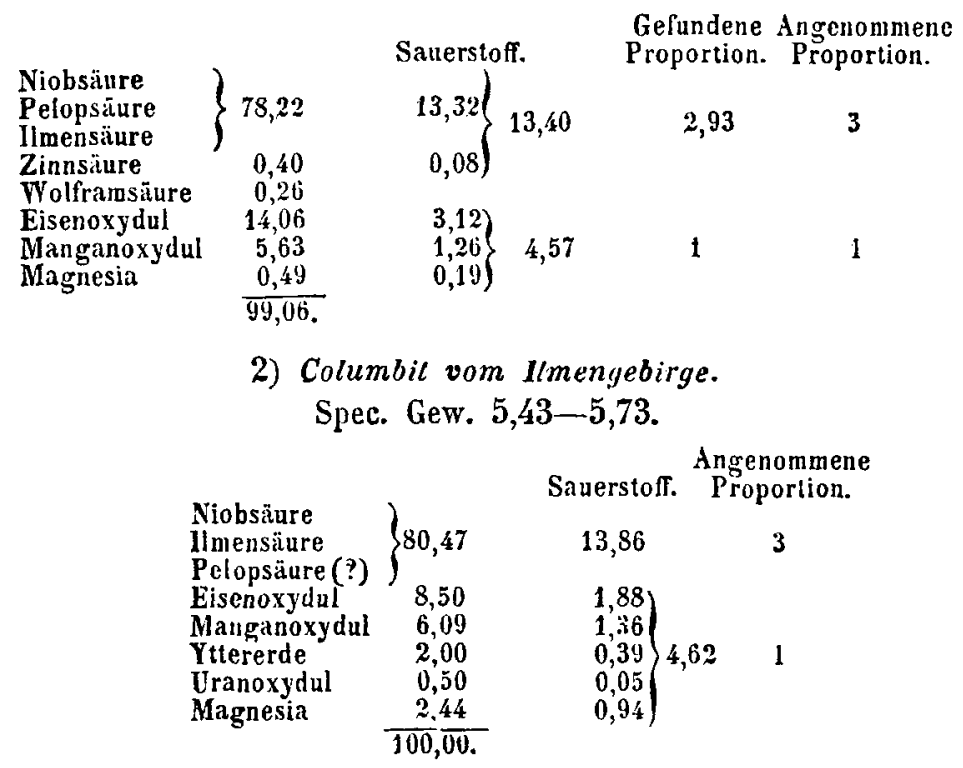


3) Columbit von Bodenmais in Baiern nach H. Rose. Spec. Gew. 6,39.

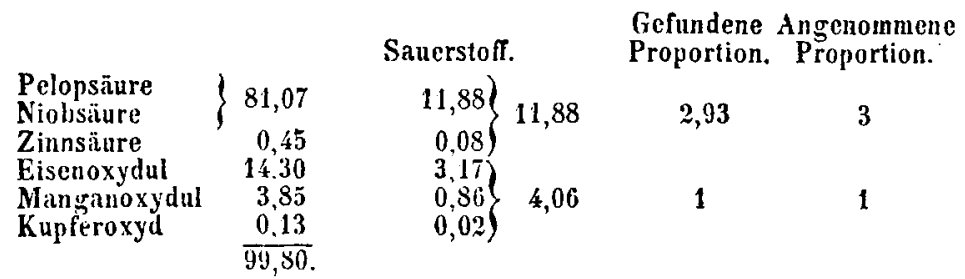

Die Formeln des Columbits bleiben also folgende:

Amerikanischer und ilmenscher Columbit.

$$
\begin{gathered}
\dot{\mathrm{R}}_{2}\left\{\begin{array}{l}
\ddot{\mathrm{N}}_{3} \\
\ddot{\mathrm{P}_{\mathrm{p}_{3}}} \\
\ddot{\mathrm{S}}_{\mathrm{i}_{3}} .
\end{array}\right. \\
\text { Bairischer Columbit. } \\
\dot{\mathrm{R}}_{2}\left\{\begin{array}{l}
\ddot{\mathrm{P}}_{\mathrm{p}_{3}} \\
\ddot{\mathrm{N}} \mathrm{b}_{3}
\end{array}\right.
\end{gathered}
$$

2) Ueber die Zusammensetzung des Ylleroilmenils und $\mathbf{S} a-$ marskils, so wie über Niobium, pelopium und Ilmenium.

Die Geschichte des Ytteroilmenits und Samarskits, so wie der in diesen Hineralien enthaltenen tantalähnlichen Substanzen fängt hereits an etwas verwickelt zu werden, weshalb es nicht überflüssig sein dürfte, sie zu rekapituliren.

Im llmengebirge finden sich, besonders an zwei Punkten, nämlich auf der Uranotantal-Grube, zusammen mit Aeschynit und auf der Columbit-Grube, zusammen mit Columbit und Monacit, noch andere Mineralien, theils in Kornern, theils in Krystallen von der Form des Columbits und hảufig mit letzteren verwachsen. Diese Mineralien sind ausgezeichnet durch ihren glatten, glänzenden, glasartigen Brucb, während die ilmenschen Columbite, mit denen sie nur verwechselt werden könnten, stets matte, unebene, ins Körnige geneigte Bruchflächen zeigen.

Eines dieser Mineralien wurde zucrst von G. Rose be- 
schrieben, der darin vorzugsweise Tantalsäure und Uranoxydul zu finden glaubte und es deshalb Uranotantal nannte.

Spăter erhielt ich unter dem Namen von Uranotantal ein Mineral, welches $z$ war alle die von Rose vom Uranotantal angegebenen äusseren Charaktere hatte, in dem sich aber, ausser den von $\mathrm{Rose}$ angegebenen Bestandtheilen, noch eine grosse Menge Yttererde vorfand. Da ausserdem die in diesem Nineral enthaltene metallische Säure alle Eigenschaften besass, die von Wöhler der Tantalsïure beigelegt worden waren, und die sonstige Zusammensetzung dicses Minerals mit der des schwedischen Ytterotantalits übereinstimmte, so hielt ich dieses Mineral ebenfalls für Ytterotantalit. - In diese Zeit fält H. Roses Entdeckung des Niobiums. Durch die Arbeit dieses Chemikers wurde es klar, dass der ăchten Tantalsäure andere Eigenschaften zukamen, als Wóhler angegeben hatte und als auch ich bereits bei den metallischen Säuren des sibirischen Ytterotantalits und des Aeschynits bemerkt hatte. Diese Säuren konnten also keine Tantolsäure sein. Bei den sogleich angestellten weiteren Versuchen ergab es sich, dass die Eigenschaften der Säure des Aeschynits im Allgemeinen mit denen übereinstimmten, die $\mathrm{R}$ os e der Niobsäure beigelegt hatte, dass aber die Săure im sibirischen Ytterotantalite wesentlich verschieden war, sowohl von der Niobsäure, als auch von der Tantalsüure, dass man sie daher als das 0xyd eines neuen Metalles betrachten müsse, welches ich Ilmenium nannte. Da also der sibirische Ytterotantalit keine Tantalsäure, sondern Ilmensäure enthielt, so gab ich diesem Minerale den Namen Ytteroilmenit.

In Folge der Entdeckung des Ilmeniums machte H. Rose bekannt, dass er im bairischen Columbite neben der Niobsäure, noch die Säure eines anderen neuen Metalles, des Pelopiums, enldeckt habe, für welches er die Priorität in Anspruch nehmen müsse. Zugleich beschrieb Ros e die Eigenschaften der Pelopsãure, so weit er sie bis dahin ausgaemittelt hatte.

Bald darauf erklärte H. Rose, dass die Ilmensäure nichts weiter sei, als ein Gemenge von Niọbäure und Wolframsäure und dass der Ytteroilmenit identisch sei mit Uranotantal, für welches Mineral er aber, weil es keine Tantalsäure, sondern Niobsảure enthalte, den Namen Samarskit vorschlug. 
Rose scheint zu dieser Ansicht besonders durch den Umstand bestimmt worden zu sein, dass es, wie ich sogleich weiter nachweisen werde, zwei verschiedene Mineralien giebt, den Samarskit und den Ytteroilmenit, die sich so älnlich sind, dass sie durch ihre äusseren Charaktere nicht unterschieden werden können. Rose hatte bisher offenbar blos Samarskit unter Händen, der in der That vorzugsweise Niobsäure enthält. Uebrigens habe ich Rose's Ansicht ïber die Natur der Ilmensäure bereits an einem anderen Orte: „Bemerkungen zu H. Roses Urlkeil über das Ilmenium", dieses Journ. XLII, 129 beleuchtet. Ich glaube bewiesen zu haben, dass an eine Identität von Ilmenium und Niobium gar nicht gedacht werden könne. Weniger klar dagegen sind die Beziehungen zwischen llmenium und Pelopium. Die allgemeinen Eigenschaften beider Stoffe haben grosse Aelinlichkeit, doch sind das spec. Gew. und das Alomgewicht der Pelopsäure viel hüher, als die der llmensāure.

Das spec. Gew. der Pelopsäure schwankt nach Rose zwischen 5,49 und 6,72, während das spec. Gew. der llmensāure $4,1-4,2$ beträgt.

Das Atomgervicht von Pelopsăure, die ich aus bairischem Columbite abgeschieden hatte und die ich durch wiederholtes Umbrystallisiren der Natronsalze so viel wie möglich von Niobsäure zu befreien suchte, betrug 1237,5. Das Atomgewicht der limensäure dagegen betrug 589,59. Ich habe aber freilich keine Bürgschaft dafür, dass die von mir dargestellte Pelopsäure ganz rein war.

Bei dieser Gelegenheit muss ich auch bemerken, dass die tantalähnliche Säure des Aeschynits und die aus dem bairischen Columbite abgeschiedene Niobsäure nur in ihren allgemeinen Eigenschaften übereinstimmen, ausserdem aber manche Verschiedenheiten zeigen.

So beträgt das spec. Gew. der Niobsäure aus Columbit nach H. Ros e $4,56-5,26$.

Die Săure des Aeschynits hat ein spec. Gew. von 3,95-4,05.

Das Natronsalz der Niobsäure aus Columbit krystallisirt in seidenglänzenden Blättern und hat grosse Aehnlichkeit mit den Krystallen des tantalsauren Natrons. 
Das Natronsalz der Säure des Aeschynits bildet kleine glasglänzende Blättchen.

Das wasserfreie Natronsalz der Niobsäure des Columbits bestand aus :

\begin{tabular}{ll} 
Niobsäure & 80,16 \\
Natron & 19,84 \\
\cline { 2 - 2 } & $100,00$.
\end{tabular}

Das Atomgew. der Niobsãure aus Columbil würde hiernach 1579,0 betragen.

Das wasserfreie Natronsalz der Säure aus Aeschynit bestand aus:

\begin{tabular}{lr} 
Silure & 78,96 \\
Natron & 21,04 \\
\cline { 2 - 2 } & $100,00$.
\end{tabular}

Das Atomgewicht der Sãure betrug 1451,53.

Das Hydrat der Sãure des Aeschynits und ihre Verbindung mit Schwefelsäure lösen sich rollständig und klar in heisser concentrirter Salzsãure.

Dieselben Verbindungen der Säure aus Columbit lösen sich zwar sehr reichlich, aber gewǒhnlich nicht vollständig und auch nicht klar in Salzsäure.

Beruhen diese Verschiedenheiten beider Sãuren auf einer Verschiedenleit ihrer Natur oder auf fremdartigen Beimengungen? Diese Frage lüsst sich noch nicht mit Sicherheit beantworten; wir müssen erst Helhoden kennen lernen, um Gemenge verschiedencr tantalähnlicher Säuren mit Schärfe trennen zu können. Wenn man aber berüclisichtigt, dass das spec. Gew. der Sãure aus Columbit viel hüher ist, als das der Săure aus Aeschynit; dass ilrr Natronsalz grosse Aehnlichkeit hat mit dem Natronsalze der Tantalsäure; dass ihr Atomgewicht höher ist als das der Säure aus Aeschynit und dem der Tantalsäure sehr nahe kommt; dass sie sich nicht klar in Salzsäure löst: so kann man sich der Vermuthung nicht erwehren, dass diese abweichenden Eigenschaften der Niobsäure aus Columbit durch eine Beimengung von Tantalsäure bewirkt werden. Wenn aber schon die Niobsãure aus dem bairischen Columbite Tantalsäure enthalten sollte, so müsste diess um so mehr der Fall sein mit der aus diesem Mlinerale durch Sublimation des Chlorids ahgeschiedenen Pelopsäure, da das Tantalchlorid ebenfalls sehr flüchtig ist. 
Ich kehre jetzt zum Ytteroilmenit und Samarskit zurück. Gegen Rose's Angabe, dass diese Mincralien identisch seien, habe ich schon angcfülirt, dass man unter den liineralien mit glasigem Bruche vou oben angegrbenen Fundorten, Krystalle antreffe, deren metallische Säure in Salzsäure unlüslich sei; diese Krystalle wären Ytteroilmenit und enthielten Ilmensäure. Andere kirgstalle dagegen enthielten in Salzsäure lüsliche Niobsäure; diese hrystalle wären Samarshit.

Die Beschaffenheit und Zusammensetzung des Ytteroilmenits babe ich bereits an andern 0rten wiederholt beschrieben. Doch werde ich der Vollstïndigkicit wegen seine Zusammensetzung hier nochmals angeben. Der Ytteruilmenit bestand in 2 Versuchen aus :

\begin{tabular}{|c|c|c|c|c|c|c|}
\hline \multirow{3}{*}{$\left.\begin{array}{l}\text { Ilmensśure } \\
\text { Titausiüe }\end{array}\right\}$} & $\begin{array}{l}\text { Spec. Gew. } \\
5,398 \text {. }\end{array}$ & Spec. Gew. & \multicolumn{2}{|c|}{ Saucrstoff. } & \multicolumn{2}{|c|}{$\begin{array}{l}\text { Gefund. Ange } \\
\text { Proport. Prop }\end{array}$} \\
\hline & & Iil $5 \pi, 813$ & 11,72 & & & \\
\hline & 01,30 & $\ddot{\mathbf{T}} \mathbf{i} \quad \check{\mathbf{s}}, 90 \mathrm{t}$ & $2,34\}$ & 14,00 & 2,01 & \\
\hline $\begin{array}{l}\text { Yttererde*) } \\
\text { Eisenoxydul }\end{array}$ & $\begin{array}{r}19,74 \\
7 \geq 3\end{array}$ & $\begin{array}{r}18,302 \\
13,613\end{array}$ & $\begin{array}{l}3,26 \\
3,00\end{array}$ & & & \\
\hline Mianganoxydul & 1,00 & 0,310 & 0,06 & & & \\
\hline $\begin{array}{l}\text { Kilkerde } \\
\text { Uranoxydnl }\end{array}$ & $\begin{array}{r}2,08 \\
\tilde{5}, 64\end{array}$ & $\begin{array}{l}0,500 \\
1,869\end{array}$ & $\begin{array}{l}0,14 \\
0,20\end{array}$ & $0, \sqrt{ }{ }^{\prime}$ & 1,00 & \\
\hline$\left(\dot{\mathrm{Z}}_{\mathrm{r}}, \dot{\mathrm{C}}, \dot{\mathrm{L}} \mathrm{r}\right)$ & 1,50 & 2,273 & $0,33)$ & & & \\
\hline Thivectiuse & 1,66 & - & & & & \\
\hline & 100,18 & $100,581$. & & & & \\
\hline
\end{tabular}

Die Formel des Yutteroilmenits ist also $=\dot{\mathrm{R}}\left\{\begin{array}{l}\ddot{\text { Il }} \\ \ddot{T} \text { i. }\end{array}\right.$

Iüurzlich habe ich auch den Samarskit untersucht. Das Resultat meiner Analyse stimmt im Wesentlichen mit den unter Rose's Leitung angestellten Untersuchungen dieses Minerals überein.

Der Krystall von Samarskit, der zu dieser Analyse diente, war $150 \mathrm{Gran}$ sclwwer. Von Columbit war an diesem Stücke nichts zu sehen.

Von Krystallflichen waren vorhanden: Die vorwaltende Querfläche $\boldsymbol{M}$, die Längsfiäche $\boldsymbol{P}$ und in der Endigung ein Oktaëder, das sich nicht näher bestimmen liess, wabrscheinlich 3 b. Fig. 3, s. Seite 165 .

*) Atomgew. der Yttererde $=\tilde{j} f \mathfrak{i}, \mathrm{J}$. 
Farbe schwarz; äusserlich mit einer graubraunen Kruste überzogen. Bruch muschlich, glatt, glänzeud, glasartig. Undurchsichtig. Härte zwischen Apatit und Feldspath. Spec. Gew. 5,64.

Löthrohr-Verhalten: das bekannte iles Uranotantals.

Die Zerlegung des Minerals wurde durch wiederholtes Schmelzen mit saurem schwefelsaurem Kali ausgeführt.

Der ausgewaschenen schwelelsauren Niobsäure liess sich weder durch Ammoniak noch durch hydrothionsaures Ammoniak Wolframsäure oder Zinnoxyd entziehen.

Ammoniak schlug die von der Schwefelsäure gelösten Stoffe fast vollstāndig nieder.

In der von diesem Niederschlage abfiltrirten Flüssigkeit zeigte lydrothionsaures Ammoniak Spuren von Kupfer an. Kleesaures Ammoniak gab lieine Spur eines Niederschlags. Phosphorsaures Ammoniak fällte eine sihr geringe Menge Hagnesia.

Der Ammoniak-Niederschlag wurde in Salzsiaure gelöst und diese Lősung mit kohlensaurem Baryt in Ueberschuss digerirt.

Hierdurch wurde ein Gemenge von Uranoxyd und Eisenoxyd, verunreinigt durch Ceroxyd und Yttererde niedergeschlagen. In der Lossung fand sich manganlaaltige Yttererde mit geringen Nengen Lanthanerde. Man schlug sie durch Anmoniak nieder.

Das Gemenge aus kohlensaurem Baryt, Uranoxyd und Eisenoxyd wurde in Salzsäure gelöst, der Baryt durch Schwefelsäure gefällt und das Uranoxyd vom Eisenoxyd durch kohlensames Ammoniak geschieden. Das in den kohlensauren Ammoniak ungelüste unreine Eisenoxyd, Ceroxyd u. s. w. wurde mit der vom kohlensauren Baryt nicht abgeschiedenen Yttererde und Lanthanerde vereinigt und anf bekannte Weise getrennt.

Zur Ausmittelung der Natur der in dem Samarskite enthaltenen tantalahnlichen Säure wurden noch folgende Versucht angestellt.

Die noch feuchte schwefelsaure Säure, wie sie durch $\mathbf{Z u}-$ sammenschmelzen von Samarskit mit saurem schwefelsaurem Kali erhalten wird, lōste sich beim Erwärmen mit concentrirter Salzsäure sehr reichlich, wiewohl nicht vollständig auf.

Diese salzsaure Lösung gab mit Zink anfänglich eine schmutzig blaue, später braune Färbung.

Die Säure wurde beim Glühen stark gelb.

Journ. f. praks. Chemie. L. 3. 
Das Natronsalz krystallisirte in kleinen Tafeln und Scluuppen. Das wasserfreie Salz bestand aus:

\begin{tabular}{lr} 
Săure & 78,26 \\
Natron & 21,74 \\
\cline { 2 - 2 } & $100,00$.
\end{tabular}

Das Alomgericht der Säure betrug also 1402,5.

Das spec. Gew. der im liohlenfeuer geglühten pulverförmigen Säure betruỡ 4,43 .

Das Natronsalz gab mit Kaliumeisencyaü̈r und Salzsäure und nit Galläpfeltinktur und Salzsäure ziegelrothe Niederschläge.

Aus diesen Versuchen lolgere ich, dass die Säure des Samarskits grüsstentheils aus Niobsiure bestelit, der aber eine geringe Menge einer andern tantalähnlichen Säure, wahrscheinlich von Ilmensäure beigemengt sein musste, weil sich dic Säure nicht ganz klar in Salzsăurc löste, und weil ihr Atomgewicht etwas niedriger war, als bei der Säure des Aeschynits.

Als Resultate meiner Analyse des Samarskits erhielt ich:

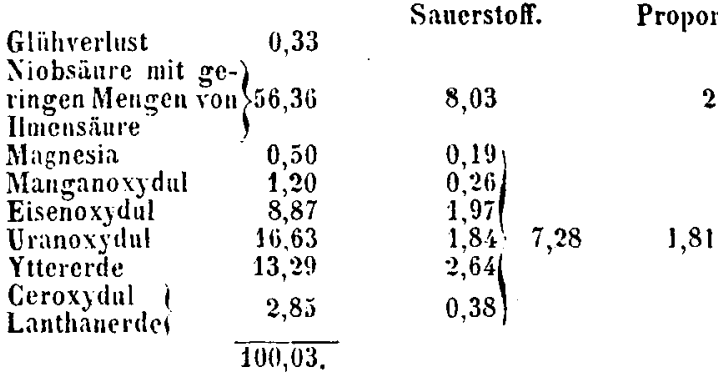

Gefundene

Hicraus croicht sich, dass der von mir untersuchle Samarskit im Wesentlichen aus einer Verbindung $=\dot{\mathrm{R}}_{2} \ddot{\mathrm{N}} \mathrm{b}$ hestand.

Auch die unter Rose's Leitung von Herrn v. Peret $z$ untersuchten Samarskite führen ganz genau zu dieser Formel, wenn man der Berechnung das von mir schon früher angegebene Atomgervicht des Niobsäure aus deschynit zu Grunde legt.

Diese Samarskite waren mit Columbit verwachsen und stammten daher aus der Columbit-Grube. Spec. Gew. 5,61-5,62. 
Sio bestanden aus:

\begin{tabular}{|c|c|c|c|c|c|c|}
\hline \multirow{5}{*}{$\begin{array}{l}\text { Niobsäure } \\
\text { Magnesia } \\
\text { Kalkerde } \\
\text { Eisenoxyda }\end{array}$} & & & 3. & Mittel. & Sauerstoff. & $\begin{array}{l}\text { Gefund. } \\
\text { Proport. }\end{array}$ \\
\hline & & & & & & 2,0 \\
\hline & & & & & & \\
\hline & & & & & & \\
\hline & 15,4 & 15,90 & 15,94 & 15,76 & $3,50>7,72$ & 2,0 \\
\hline \multirow[t]{2}{*}{$\begin{array}{l}\text { Uranoxyd } \\
\text { Yttererde }\end{array}$} & $\begin{array}{r}14,16 \\
9,15\end{array}$ & $\begin{array}{l}16,70 \\
11,04\end{array}$ & $\begin{array}{r}16,77 \\
8,36\end{array}$ & U் $\begin{array}{r}15,88 \\
9,51\end{array}$ & \multirow[t]{2}{*}{$\begin{array}{l}1,67 \\
1,89\end{array}$} & \\
\hline & & & & & & \\
\hline \multicolumn{7}{|c|}{$\begin{array}{l}\text { Die Formel dieser Samarskite ist also ebenfalls }=\dot{\mathrm{R}}_{2} \ddot{\mathrm{N}} \mathrm{b} \text {. } \\
\text { Aus diesen Untersuchungen geht also ganz entschieden her- } \\
\text { dass der Ytteroilmenit und der Samarskit ganz verschiedene } \\
\text { eralien sind. }\end{array}$} \\
\hline \multicolumn{7}{|c|}{$\begin{array}{l}\text { Sie enthalten ganz verschiedene metallische Säuren; ilır Ge- } \\
\text { an Uranoxydul und Yttererde ist beträchtlich abweichend; } \\
\text { ihre stöchiometrische Constilution führt zu ganz verschie- } \\
\text { en Formeln. Der Ytteroilmenit ist ein neutrales Salz mit } \\
\text { chen Atomen Säure und Basis, der Samarshit dagegen ein } \\
\text { sches Salz mit } 2 \text { Atomen Basis und } 1 \text { Atom Saure. }\end{array}$} \\
\hline
\end{tabular}

3) Ueber die Homöomorphie von Columbit, Ytteroilmenit, Samarskit, Mengit, Polykras, Polymignit und Wolfram.

Die Uebereinstimmung der Formen von Wolfram, Columbit, Ytteroilmenit und Samarskit ist bereits anerkannt, weshalb ich mich auch nicht weiter bei diesen Mineralien aufzuhalten brauche. Ich werde mich daher hier nur mit den Formen von Mengit, Polykras und Polymignit beschäftigen.

\section{$M e n g i t$.}

Der Mengit findet sich, wiewohl sehr sparsam und immer nur in einzelnen kleinen Krystallen, auf mehreren Gruben in der Nähe des Ilmensees bei Miask.

Ich besitze zwei Exemplare dieses seltenen Minerals. Der eine Krystall ist anfgewachsen auf fleischrohem Feldspath. $\mathrm{Er}$ fand sich zusammen mit Aeschynit in der Entfernung einiger Schritte von der Topas-Grube No. 5.

Der zweite Krystall ist aufgewachsen auf Amazonenstein. Er fand sich auf einem Gange von Schriftgranit 1 Werst ostlich von der Topas-Grube No. $\mathbf{5}$. 
Die Form dieser lirsstalle zeigt Fig. 4.

Sie stimmt ganz mit der Beschreibung aherein, die Brooke von dem Mengit gegeben hat. Brooke beschreibt sie nämlich wie folgt:

Krystall-System ein- und einaxig.

Vertikale rhombische Prismen von $136^{\circ} 20^{\prime}$ mit der Längs1läche und einem anderen rhombischen Prisma, welches zu jenem unter $140^{\circ} 14^{\prime}$ geneigt ist. In der Endigung erscheint ein Rhomben-Olitaèder, dessen Flächen auf denen des rhombischen Prismas von $136^{\circ} 20^{\prime}$ gerade aufgesetzt sind, in seinen Endkanten aber tinter $150^{\circ} 32^{\prime}$ und $101^{\circ} 10^{\prime}$ geneigt ist.

Breithaupt hat bereits darauf aufmerksam gemacht, dass cliese Winkel mit denen des Columbils übereinstimmen. Denn nimmt man die von $B$ rooke als Längsfläche bezeichnete Fläche als Querfläche, so beträgt der Winkel der beiden vertikalen Prismen $43^{\circ} 20^{\prime}$ und $100^{\circ} 28^{\prime}$. Das Oktaëder dagegen erhält die Winkel $150^{\circ} 32^{\prime}, 101^{\circ} 10^{\prime}, 86^{\circ} 21^{\prime}$.

Diese Abmessungen stimmen sehr genau mit denen des Columbits überein, bei dem betragen:

Vertikales Prisma $d\left(a: \frac{1}{3} b: \infty c\right)=43^{\circ} 48^{\prime}$,

Vertikales Prisma $f(a: b: \infty c)=100^{\circ} 40^{\prime}-100^{\circ} 16^{\prime}$,

Phomben-Olitaëder $3 b(a: 36: c)=150^{\circ} 17^{\prime}, 100^{\circ} 49^{\prime}, 86^{\circ} 25^{\prime}$.

Rose hat als wesentliclue Bestandtheile des Mengits angeyeben: Titansäure, Zirkonerde und Eisenoxydul.

Das duftreten von Zirkonerde in einem Ninerale, welches die Form des Columbits hat, in welchem letzeren leine dreiatomigen Basen rorkommen, ist allerdings sehr auffallend. Wir werten aber sogleich nachweisen, dass noch zwei andere Mineralien, in denen Zirkonerde vorkommt, die Form von Columbit haben. Es sicht demmach so aus, als wenn die Zirkonerde einatomige Basen vertreten könne, mithin wahrscheinlich selbst nur ein Atom Sauerstoff enthalten dürfte. 


$$
\text { polykras. }
$$

Scheerer hat folgende Abmessungen des Pulykrases gegeben:

krystallsystem ein- und einaxig.

$$
\begin{aligned}
& a: b=90^{\circ} \\
& p: p=140^{\circ} \\
& P: b=104^{\circ} \\
& P: P=152^{\circ} .
\end{aligned}
$$

H a u $\mathrm{m}$ an $n$ hat aus diesen Abmessungen folgende Formen berechnet:

Rhombenoktaëder $\boldsymbol{P}=152^{\circ} 0^{\prime}, 9^{\circ} 2^{\prime}, 96^{\circ} 41^{\prime}$, Vertikales Prisma $p=140^{\circ}$,

Querprisma $h=56^{\circ} 0^{\prime}$.

Figur 5.

Giebt man aber dem Polyliras eine andere Stcllung; betrachtet man nämlich das Prisma $p$ als Längsprisma und die Quernliche $a$ als Basis, so erhält man Winkel, die, mit Ausnahme des Oktaëders, sehr nahe mit denen des Columbits übereinstimmen. Es betragen dann namblich:

Olitaëder $P=152^{\circ} 0^{\prime}, 96^{\circ} 41^{\prime}, 90^{\circ} 2^{\prime}$,

Querprisma $h=124^{\circ}$,

Läugsprisma $p=140^{\circ}$.

Beim Columbit betragen aber die Winkel:

des Oktaëders $3 b(P)=(a: 3 b: c)=150^{\circ} \quad 17^{\prime}, 100^{\circ} 49^{\prime}$ $86^{\circ} 52^{\prime}$,

des Querprismas $o(h)=\left(a: \infty b: \frac{1}{2} c\right)=123^{\circ} 50^{\prime}$ bis $125^{\circ} 20^{\prime}$,

des Längsprismas $v(p)=\left(\infty a: 1 \frac{2}{3} b: c\right)=139^{\circ} 6^{\prime}$.

Als wesentliche Bestandtheile des Polykrases hat Scheerer gefunden: Tantalsäure(?), Titansäure, Zirkonerde, Yttererde, Eisenoxydul, Uranoxydul uni Ceroxydul.

$$
\text { P o } l y m i g n i \ell \text {. }
$$

Auch die Form des Polymignits stimmt wahrscheinlich mit der des Columbits überein, da nach den Messungen von G. Ros c an diesem Minerale 3 Prismen vorkommen, von $70^{\circ} 50^{\prime}$, $109^{\circ} 10^{\prime}$ und $140^{\circ} 51^{\circ}$, deren Winkel fast vollkommen mit den Quarzprismen des Columbits übereinstimmen. Diese betragen nämlich: 


$$
\begin{aligned}
& m=\left(a: \infty \quad b: 1 \frac{1}{3} c\right)=70^{\circ} 50^{\prime} \\
& n=\left(a: \infty b: \frac{2}{3} c\right)=109^{\circ} 35^{\prime} \\
& p=\left(a: \infty b: \frac{1}{3} c\right)=141^{\circ} 8^{\prime}
\end{aligned}
$$

Aber die Abmessungen des Oktaëders des Polymignits $=$ $136^{\circ} 28^{\prime}, 116^{\circ} 22^{\prime}, 80^{\circ} 26^{\prime}$, lassen sich nicht ungezwungen auf die Axen-Verhältnisse des Columbits übertragen. Dieses Oktaëder wũrde dann die Formel $=\left(a: 1 \frac{5}{6} b: 1 \frac{2}{3} c\right)$ erlıalten, welche Verhältnisse wenig Wahrscheinlichkcit für sich haben. Es muss daher weiteren Untersuchungen überlassen bleiben, um zu entscheiden, wie es sich mit diesem Oktaëler verhält.

Auch die stüchiometrische Constitution des Polymignits lässt sich aus der Inalyse dieses llinerals von Berzelius nicht erkennen, besonders wegen des nicht unbeträchtlichen Verlustes, der wahrscheinlich aus alkalischen Substanzen bestand.

Wir wissen daher nur, dass sich im Polymignit als Hauptbestandtheile vorfinden: Titansäure, Zirkonerde, Eisenoxydul und Yttererde, dass also die Zusammensetzung des Polymignits grosse Aehnlicbkeit hat mit der des Mengits.

In der Form des Columbits krystallisiren also sehr verschiedene Verbindungen. Dieselben sind:

1) Mengit $=\dot{\mathrm{Z}} \mathrm{r}, \dot{\mathrm{F}} \mathrm{e}, \ddot{\mathrm{T}} \mathrm{i}$.

2) Polymignit $=\dot{\mathrm{Z}} \mathrm{r}, \dot{\mathrm{F}} \mathrm{e}, \dot{\mathrm{X}}, \ddot{\mathrm{T}} \mathrm{i}$.

3) Polykras $=\dot{Z}_{\mathrm{i}}, \dot{\mathrm{F}} \mathrm{e}, \dot{\mathrm{U}}, \dot{\mathrm{C} e}, \ddot{\mathrm{T}} \mathrm{i}, \ddot{\mathrm{T}} \mathrm{a}(?)$. $\dot{\mathrm{U}}_{2}$

4) Samarskit $\left.=\underset{\dot{\mathrm{F}}_{2}}{\dot{\mathrm{Y}}_{2}}\right\}$ Nib.

5) Ytteroilmenit $\left.=\begin{array}{l}\dot{\mathrm{Y}} \\ \dot{\mathrm{F} e} \\ \dot{\mathrm{U}}\end{array}\right\}\left\{\begin{array}{l}\ddot{\mathrm{I}} \\ \ddot{\mathrm{T}} \mathrm{i}\end{array}\right.$

6) Columbit $\left.=\begin{array}{l}\dot{\mathrm{F}} \mathrm{e}_{2} \\ \dot{\mathrm{I}} \mathrm{n}_{2}\end{array}\right\}\left\{\begin{array}{l}\ddot{\mathrm{N}} \mathrm{b}_{3} \\ \ddot{\mathrm{P}}_{\mathrm{p}_{3}} \\ \ddot{\mathrm{I}}_{3}\end{array}\right.$

7) Wolfram $\left.=\begin{array}{c}\dot{\mathrm{F} e} \\ \dot{\mathrm{MI} n}\end{array}\right\} \ddot{\mathrm{W}}$.

Auch die Winkel des Brookits stimmen sehr nahe mit de- 
nen des Columbits überein. Die Unterschiede sind nicht grüsser als sie hänfig bei anerkannt isomorphen oder heteromeren Körpern gefunden werden.

Ehe wir die in der Form des Columbits krystallisirenden Mineralien verlassen, müssen wir noch einen Blick auf die so auffallende Erscheinung werfen, dass so verschiedenartig zusammengesetzte Verbindungen dieselbe Form baben. Wir haben dabei besonders die Frage zu beantworten: welchen Autheil hat an dieser Erscheinung die Isomorphie und welchen die Heteromerie?

Als eine Folge von Isomorphie betrachte ich die gegenseitige Vertretung von Niob-Pelop- Ilmen- und Titansäture.

Mengit ist ein Mineral, welches als elektronegativen Bestandtheil vorzugsweise Titansāure enthält und doch stimmt seine Form vollkommen mit der des Columbits überein, in welchem vorzugsweise Niob- Pelop- und llinensãure vorkommen. Dieselbe Erscheinung wiederholt sich beim Perowskit und Pyrochlor.

Ausserdem geht aus der Zusammensetzung der Natronsalze der tantaăhnlichen Säuren hervor, dass in diesen Säuren 2 Atome Sauerstoff enthalten sind, dass sie also dieselbe stöchiometrische Constitution wie die Titansäure haben.

Es kann daher gar keinem Zweifel unterliegen, dass die Titansäure und die tantalähnlichen Sāuren isomorph seien. Als eine Folge von Isomorphie betrachte ich ferner das Auftreten von Zirkonerde in Mineralien von der Form des Columbits und Pyrochlors. Da in diesen Mineralien keine dreiatomigen Basen vorkommen, so muss die Zirkonerde in den erwähnten Fällen einatomige Basen ersetzen; sie kann daher nur ein Atom Sauerstoff enthalten. In der That lässt sich, seitdem man auch in der Beryllerde nur ein Atom Sauerstoff annimmt, die Zusammensetzung der Zirkonerde nach der Formel $\dddot{Z}_{r}$ gar nicht mehr rechtfertigen. Denn welche Analogien existiren wohl zwischen Zirkonerde und Thonerde? Und in welchen Mineralien würden wohl Thonerde oder andere dreiatomige Basen durch Zirkonerde oder umgekehrt vertreten? Dagegen bildet Zirkonerde eine natürliche Reihe mit Beryllerde, Yttererde, Lanthanerde und Thorerde und 
vertritt auch, wie wir eben sahen, die eine oder die andere dieser Basen in den Mineralien von der Form des Columbits, zuweilen auch, wie wir später sehen werden, im Pyrochlor.

In das Gebiet der Heteromerie gehört dagegen die Erscheinung, dass drei salzălıbliche Verbindungen von verschiedener Săttigung wie Samarskit $=\dot{\mathrm{R}}_{2} \ddot{\mathrm{N}} b$, Ytteroilmenit $=\mathrm{K}$ ïl und Columbit $=\dot{R}_{2}\left\{\begin{array}{l}\ddot{\mathrm{N}} \mathrm{b}_{3} \\ \ddot{\mathrm{P}}_{\mathrm{p}_{3}} \\ \ddot{\mathrm{i}}_{3}\end{array}\right.$, in derselben Form krỵstallisiren.

Ferner der Limstand, dass Wolfram $=\dot{R} \ddot{W}$ dieselbe Form hat wie Columbit.

Letztere Erscheinung hat bereits zu ben rerschiedensten Deutungen Veranlassung gegeben. Man glaubte darin den Beweis zu finden; entweder:

dass die tantalähnlichen Süuren wie die Wolframsüure zusam mengesetzt wiren, mithin 3 Atome Saterstoff enthalten mūssten, wodurch man aber in Widerspruch mit der Isomorphie dieser Säuren mit der Titansäure gerielh; oder dass der Wolfram leine Wolframsüure sondern Wolframoxyd $=\ddot{\mathbb{V}}$ enthalte.

Letztere Ansicht ist aber bereits durch die neuesten sorgfältigsten Untersuchungen von Ebelmen, Rammelsberg etc. willerlegrt.

Hierzu muss ich namentlich noch bemerken, dass sich auch die zweite Form der natürlichen neutralen Verbindungen der Wolframsäure, nämlich die Form des Scheelspaths und Scheelbleierzes, bei den Tantalaten wiederfindet. Der Fergusonit hat nämlich, wie Bre ith a u t nachgewiesen hat, die Form des Scheelspaths. Der Vertheidiger der Ansicht, dass der Wolliram Wolframoxyd enthalte, müsste dann auch annehmen, dass der Scheelspath und das Scheelhleierz Wolframoxyd und das Gelbbleierz Molybdanoxyd enthalten; Ansichten die wohl keine Anhänger finden werden.

Die gleiche Form von Wolfram und Columbit und von Scheelspath und Fergusonit beruht demnach nicht auf Isomorphie sondern auf Heteromerie. Aehnliche Erscheinungen kommen auch bei der Verbindung anderer Säuren von ganz verschiedener stöchiometrischer Constitution vor. Ich erinnere nur an 
die Gleichheit. der Form von Kalisalpeter und Arragonit; von Natronsalpeter und Kalkspath; von Vanadinblejerz, Grünbleierz und Apatit; von Chrysolith und Chrysoberyll. Wenn aber beteromere Moleküle Vervandschaft zu einander haben, so können sie auch zusammen krystallisiren, ihre stōchiometrische Constitution mag sein, welche sie wolle. An Beispielen, welche diess beweisen, fehlt es nicht. Ich erwähne hier nur das Zusammenkrystallisiren von:

Rubellit und Schürl;

Titaneisen und Eisenglanz;

Monazit und Monazitoïd;

Aspasiolith und Cordierit;

Vanadinbleierz und Grünbleierz;

Bucklandit und Zoisit;

Ytteroilmenit, Samarshit und Columbit;

Magneteisen und Granat;

Orthit und Epidot;

Skolezit und Natrolith;

die Feldspathe, Glimmer, Skapolithe u. s. w.

\section{Ueber die Zusammenselsung der Pyrochlore.}

Mit dem Namen Pyrochlor bezeichnet man Mineralien von verschiedener Hischung. Sie krystallisiren aber alle in regulïren Oktaēdern und entlıalten alle tantalähnliche Substanzen als wesentliche Bestandheile. Die Pyrochlore bilden also eine scharf charakterisirte Gruppe in der Familie der Tantalerze.

Die wesentlichen Unterschiede der verschiedenen Arten von Pyrochlor bestehen aber darin, dass die einen weder Wasser noch Fluor, andere Wasser und kein Fluor und noch andere Fluor und kein Wasser enthalten.

Ich werde die ersteren Mikrolith, die zweiten Hydrochlor und die dritten Fluochlor nennen.

\section{Mikrolith.}

Der Mikrolith findet sich zu Cliesterfield in Massachusets in Nordamerika, auf einem Albit-Gange zusammen mit Rubellit und grünem Turmalin. 
Er bildet kleine Oktaėder von gelblicher Farbe. Spec. Gew. 4,75-5,56. Nach $S$ hepard besteht dieses Nineral aus:

\begin{tabular}{lr} 
Tantalähnlicher Säure & 75,40 \\
Kalk & 14,84 \\
Yttererde t & 7,42 \\
Uranoxyduly & Spur \\
Wolframsäure & 2,04 \\
Wasser & $\frac{2,4}{}$ \\
\cline { 2 - 2 } & $99,70$.
\end{tabular}

Zum Mikrolith gehört offenbar auch der von Hayes untersuchte Pyrochlor von Fredrikswärn. Der'selbe bestand aus:

\begin{tabular}{lr} 
Tantalähnlicher Säure & 59,00 \\
Titansiüure & $\mathbf{1 8 , 3 3}$ \\
Kalkerde & 16,73 \\
Uranoxydul & \\
$\begin{array}{l}\text { Eisenoxydul } \\
\text { Manganoxydul }\end{array}$ & \\
$\begin{array}{l}\text { Natron } \\
\text { Glühverlust }\end{array}$ & $\mathbf{0 , 7 0}$ \\
& $\mathbf{5 , 6 3}$ \\
& 0,80 \\
\hline $\mathbf{1 0 1 , 1 3 .}$
\end{tabular}

Zu dieser Analyse ist zu bemerken, dass der Gehalt an Titansauure offenbar zu hoch angegeben ist. In diesen 18,33 p.C. Titansăure mögen noch die $\mathbf{9 , 0}$ p. C. Yttererde und Ceroxydul enthalten gewesen sein, die Wöhler im Pyrochlor von Fredrikswärn angegeben hat.

Die stöchiometrische Constitution des Mlikroliths lässt sich aus diesen Analysen nicht berechnen, wei' die Natur der in diesem Minerale vorkommenden tantalähnlichen Säure noch nicht ausgemittelt ist. Es ist aber sehr wahrscheinlich, dass der Mikrolith dieselbe stochiometrische Constitution hat, wie der Perowskit, weil beide Mineralien gleiche Form haben, und ihre Bestandtheile isomorph sind. Hiernach würde der Hikrolith nach der Formel $\dot{\mathbf{R}} \ddot{\mathrm{T}} \mathrm{a}($ ?) zusammengesetzt sein.

\section{Hydrochlor.}

Zum Hydrochlor gehören die von Wöhler untersuchten Pyrochlore von Brevig und Fredrikswärn in Norwegen.

Dieselben bestanden aus: 
Brewig.

(Mit Thorit. Spec. Fredrikswärn.

Metallische Säuren $\left.\begin{array}{l}\stackrel{\operatorname{Ta}}{\mathrm{T}(?)} \\ \mathrm{Ti}\end{array}\right\}$

Gew. 3,802.) (Spec. Gew. 4,20.)

Zinnoxyd

Thorerde ?

Ceroxyd ;

Uranoxydul

Eisenoxydul

Manganoxydul

Kalkerde

Alkalien

Wasser

$\begin{array}{cc}67,021 & 62,75 \\ - & 0,61 \\ 5,159 & \dddot{\mathrm{Ce}} 6,80 \\ 4,601 & 5,18 \\ 1,329 & 2,16 \\ 1,688 & 2,70 \\ 9,877 & 12,85 \\ \text { geringe Menge } & \text { geringe Menge } \\ \underset{7,059}{96,734} & -\frac{4,20}{97,25} .\end{array}$

Ueber die stōchiometrische Constitution des Hydrochlors lässt sich ebenfalls bis jetzt nichts Sicheres sagen, da weder die Natur der in diesem Minerale vorkommenden tantalähnlichen Săuren, noch die Quantität der Titansäure und der Alkalien bestimmt wurde. Es ist aber sehr wahrscheinlich, dass der Hydrocblor Mikrolith mit 1 Atom Wasser sei. Hier hätten wir dann wieder dieselbe Erscheinung, auf die ich schon bei verschiedenen Gelegenheiten aufmerksam gemacht babe, nämlich, dass Hydrate häufig dieselbe Form haben wie wasserfreie Verbindungen. Ich erinnere an: Lepolith und Lindsayit; Allanit und Ortbit; Chrysolith, Villarsit und Serpentin; Oerstedtit, Malakon und Zirkon; u. s. w.

\section{Fluochlor.}

Dieses Mineral findet sich an mehreren Stellen des llmenGebirges bei Miask. Es wurde zuerst von Wöhler und später von mir untersucht.

Nach Wóhler bestand ein Pyrochlor von Miask, mit einem spec. Gewicht $=4,32$ aus:

$\begin{array}{lr}\begin{array}{lr}\text { Tantalähnliche Säure } \\ \text { Titansiure }\end{array} & 67,370 \\ \text { Thorerde } & \\ \text { Ceroxyd } & 13,152 \\ \text { Kalkerde } & 10,984 \\ \text { Yttererde } & 0,808 \\ \text { Eisenoxydul } & 1,285 \\ \text { Manganoxydul } & 0,146 \\ \text { Natrium } & \mathbf{3 , 9 3 0} \\ \text { Fluor } & 3,233 \\ \text { Wasser } & 1,160 \\ & \mathbf{1 0 2 , 0 7 4} .\end{array}$


Nach meinen Versuchen dagegen bestind ein Pyrochlor von Mliask mit einem spec. Gew. $=4,203$ aus:

\begin{tabular}{lrr} 
Niobsäure & \multicolumn{3}{c}{ Sauerstoff. } \\
Ilmensïure & 62,25 & 11,43 \\
Titansäure & 2,23 & 0,87
\end{tabular}$\quad 12,30$

Dass die in diesem Pyrochlore enthaltenen tantalähnlicben Säuren aus einem Gemenge von Niobsäure und llmensäure bestanden, schliesse ich aus dem Umstande, dass die Verbindung dieser Säure mit Schwefelsäure sich nur wenig in concentrirter Salzsäure löste; aus ihrem niedrigen spec. Gew. von 4,0; und aus ihrem geringen Atomgewicht von 1089,3. Ihr Chlorid enthielt näılich 50,3 p. C. Chlor.

Der Unterschicd dieser Analysen von Wo hller und mir besteht besonders darin, dass Wöhler im Fluochlnre von Miask Thorerde fand, ich dagegen nicht. Dagegen war in der von mir untersuchten Probe Zirkonerde enthalten, die in dem von Wóhler untersuchten Pyrochloren fehlte. Dieser letztere Umstand kann übrigens weiter nicht auffallen, da wir bei den Mineralien von der Form des Columbits sahen, dass Zirkonerde einatomige Basen vertreten kőnne. Uebrigens habe ich in einer anderen Probe von Fluochlor keine Zirkonerde wicderinden konnen; dieser Bestandtheil ist daher nicht constant.

Was die Thorerde anbelangt, so hat auf Wōhler's Veranlassung auch Herr. Dr. Ställer aus dem Prochlore eine Substanz abgeschieden, die auch Berzelius als Thorerde erkannt hat. War aber dieser Pyrochlor wirklich der Fluochlor aus der Gegend von Miask? War ihm nicht vielleicht zufallig Hydrochlor von Brevig, nämlich die Art von Pyrochlor, die mit Thorit zusammen vorkommt und deren Gehalt an Thorerde ich gar nicht bezweifelt habe, indem er mir sogar sehr wahrschein- 
lich ist, beigemengt? Dem mag übrigens sein wie ihm wolle, soviel geht aus Wöhler's eigenen Versuchen und aus den Analysen von Shepard, Hayes und mir hervor, dass die Thorerde keinen constanten Bestandtheil der Pyrochlore bilde.

Uebrigens habe ich den Fluochlor von Miask einer netuen Untersuchung unterworfen und werde auch den Gang der Analyse näher beschreiben. Die äussere Beschaffenheit der untersuchten Probe war die bekannte. Spec. Gew. 4,28.

\section{a. Bestiminung des Fluors.}

Zum feinsten Pulver zerriebener Fluochlor wurde in einer kleinen Retorte mit überschüssiger Schwefelsăure eingedampft und der Hals der Retorte während dieser Operation mit Ammoniak gesperrt. Es entwickelte sich dabei eine nicht unbeträchtliche Menge Kieselllusssäure, die von dem Ammoniak unter Abscheidung von Kieselerde absorbirt wurde. Die von der Kieselsãure abfiltrirte Flüssigkeit gab mit salzsaurem Kali einen Niederschlag von Fluorcalcium der 2,210 vom Gervichte des angewandten Minerals Fluor enthielt.

\section{b. Bestimmung der Alkalien und der Magnesia.}

Eine andere Portion Mineral wurde durch Schwefelsäure zerlegt. Die saure Masse wurde mit Wasser verdünnt, mit äberschüssigem Ammoniak und kleesaurem Ammoniak versetzt, filtrirt, die Lósung verdunstet und die Salze ausgeglüht. Es blieben hierbei schwefelsaure Salze von Kali, Natron und Magnesia zurück. Aus diesen Salzen erhielt man auf bekannte

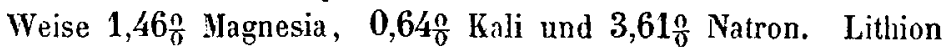
war in diesen Salzen nicht enthalten.

\section{c. Bestimmung der anderen Bestandtheile des Fluochlors.}

Eine dritte Portion Mineral wurde mit ihrer sechsfachen Menge sauren schwefelsauren Kalis in glühenden Fluss gebracht und so lange darin erhalten, bis alles Mineral gelöst war. Die erkaltete Salzmasse wurde fein zerrieben, und mit warmen Wasser behandelt und gut ausgewaschen.

Es blieb hier eine tantalähnliche Säure in Verbindung mit Schwefelsäure ungelöst, die nach starkem Glühen $60,83 \frac{0}{0}$ reimer Sảure zurückliess. 
Das Hydrat und die Verbindung dieser Säure mit Schwefelsăure lōsten sich in concentrirter Salzsäure fast vollständig auf. Das Natronsalz hatte die auussere Beschaffenheit und die Zusammensetzung des Salzes der Niobsäure aus Aeschynit.

Auch die anderen Reaktionen stimmten mit denen der Niobsäure überein. Dic tantalăhnliclıe Säure dieser Probe kann also als last reine Niobsāure betrachtet werden.

Die von der schwefelsauren Niobsäure abgelaufene Flüssigkeit gab mit Ammoniak einen reichlichen Niederschlag.

Die von dem Ammoniakniederschlag abgelaufene Flüssigkeit gab mit kleesaurem Ammoniak einen Niederschlag von kleesaurem Kalk, der 9,80\% Kalk enthielt.

Der Ammonial-Niederschlag wurde mit saurem schwefelsaurem Natron geschmolzen, die Salzmasse in warmem Wasser gelöst, die klare Lösung mit kohlensaurem Ammoniak möglichst neutralisirt und hierauf mit kleesaurem Ammoniak versetzt. Es entstand dadurch ein reichlicher Niederschlag. Derselbe enthielt Ceroxydul, Lanthanerde und Yttererde in Verbindung mit Kleesãure. In diesem Niederschlag hätte auch die Thorerde enthalten sein müssen, wenn sich solche im Fluochlore vorfänile.

Dieser Niederschlag wurde ausgeglüht und hierauf mit Schwefelsăure eingedampft. Das Salz wurde in kaltem Wasser gelöst und die Lósung mit schwefelsaurem Kali gesätligt. Dadurch fiel das bekannte weisse Doppelsalz von schwefelsaurem Ceroxydul, Lanthanerde und schwefelsaurem Kali nieder; dieses Doppelsalz hätte auch die Thorerde enthalten müssen. Man wusch es mit einer Lösung von schwefelsaurem Kali aus. In der von diesem Doppelsalz abgelaufenen Flüssigkeit gab Ammoniak nur einen sehr geringen Niederschlag von $0,94 \%$ Yttererde.

Das Doppelsalz wurde in warmem Wasser gelöst, die Lösung mit Ammoniak gefält. Es entstand dadurch ein reichiicher Niederschlag, der nach dem Glühen 15,230 eines braunen 0xydes zurückliess. Dieses Oxyd wurde von Salzsäure und Salpetersäure nur wenig angegriffen. In Schwefelsäure löste es sich vollständig mit gelber Farbe auf. Wenn man das schwefelsaure Salz in 10 Theilen kaltem Wasser löste und die Lösung erwärmte, so schied sich so viel eines dicken Niederschlags ab, dass die Flüssigkeit breiartig wurde. Sollte diese Erscheinung nicht zu den verschiedenen Gerüchten eines Thorerde-Gehaltes cerhalti- 
ger Mineralien Veranlassung gegeben haben? Dieser Niederschlag ist aber nichts anderes, als das schon früher von mir beschriebene basische schwefelsaure Ceroxyd. Denn, wenn man jenes schwefelsaure Salz zur Trockne verdampft und den Rủckstand gelind glüht, so verliert er seine gelbe Farbe und wird weiss. Das schwefelsaure Ceroxyd wird dabei zu schwefelsaurem Ceroxydul. Wenn man jetzt das Salz in 10 Theilen kalten Wasser löst und die Lösung erwärmt, so bleibt sie ganz klar und es setzt sich keine Spur von schwefelsaurer Thorerde ab. Wenn man aber die schwefelsaure Lüsung lange kochte, unter Erneterung des verdampfenilen Wassers, so bilden sich weisse Krusten von basisch schwefelsaurem Ceroxydul.

Das reducirte weisse schwefelsaure Salz, welches aus jenem $15,23 \frac{0}{0}$ braunen Oxyd dargestellt wurcle, enthielt auf 500 Theile Schwefelsăure 661 Basis.

Es liess sich also weder durch Erhitzen einer concentrirten Lösung des reducirten schwefelsauren Salzes der Masse des Fluochlors, in der die Thorerde enthalten sein musste, diese Erde abscheiden; noch war die quantitative Zusammensetzung dieses schwefelsauren Salzes der Art, dass sie einen Gehalt an Thorerde andeutete.

Die von dem Niederschlage, den kleesaures Ammoniak bewirkt hatte, und der das Ceroxydul und die Yttererde enthielt, abgelaufene Flüssigkeit wurde mit Weinsteinsäure versetzt, hierauf mit Ammoniak äbersättigt und mit hydrothionsaurem Ammoniak versetzt. Hierbei fiel Schwefeleisen, welches ein Aequivalent von 2,23응 Eisenoxydul enthielt.

Die von dem Schivefeleisen befreite Flüssigkeit wurde zur Trockne verdınstet, das Salz geglüht und nach Zusatz von überschüssiger Schwefelsäure geschmolzen. Die saure Salzmasse wurde in Wasser gelōst und durch Ammoniak niedergeschlagen. Der Niederschlag löste sich im feuchten Zustande leicht in Salzsäure. Die salzsaure Lósung hinterliess nach dem Verdunsten in der Wärme einen braunen Firniss, der keine Spur von Krystallen zeigte. Dieser Niederschlag enthielt also keine Zirkonerde. Er bestand aus Titansüure.

Als Resultat dieser Analyse des Fluochlors von Miask erhielt man also: 


\begin{tabular}{lrrr} 
& \multicolumn{3}{c}{ Sauerstoff. } \\
Niobsäure & 60,83 & 8,37 & 10,31 \\
Titansänre & $\mathbf{4 , 9 0}$ & 1,94 & 10,31 \\
Ceroxydul & 15,23 & 2,03 & \\
Lanthanerde & 15 & \\
Yttererde & 0,94 & 0,18 & \\
Eisenoxydul & 2,23 & 0,49 & 6,05 \\
Kalkerde & 9,80 & 2,80 \\
Magnesia & 1,40 & 0,55
\end{tabular}

Bei der Berechnung der Formel des Fluochlors bin ich von folgenden Ansichten ausgegangen.

Der Fluochlor nurde betrachtet als eine Verbindung der Grundmischung der Pyrochlore $\mathbf{H}$ T̈a(?) mit einer Fluor-Verbindung. Aus den gefundenen Proportionen ergab sich aber, dass diese Fluor-Verbindung kein einfaches Fluorid, sondern ein $0 x y$ fluorid sein müsse.

Denn zieht man das einfache Aequivalent des Fluors an Sauerstoff von dem Sauerstoffgehalte der gefundenen Basen $a b$, so bleibt in meiner früheren Analyse das Sauerstoff-Verhältniss von $7,35: 12,30$; in meiner späteren Analyse aber das Verhältniss von $5,90: 10,31$; Verhältnisse welche nicht mit dem von $1: 2$ übereinstimmen. Zieht man aber das doppelte Aequivalent des Fluors an Sauerstoff ab, so erhält man die Proportionen von $6,07: 12,30$ und von $4,96: 10,31$; Verhältnisse die dem von 1 : 2 sehr nahe kommen.

Die Verhältnisse der Aequivalente des Fluors zu denen der Basen sind aber $1: 4,66$ und 1:5,27. Hieraus ergiebt sich, rlass die Fluor-Verbindung zu der Verbindung der tantalähnlichen Säuren in wechselnden Verhältnissen stehe. Die Fluochlore werden daher olfenbar aus zwei verschiedenen Verbindungen gebildet, die zusammen krystallisiren können; nämlich aus:

$$
\begin{aligned}
& \dot{\mathrm{R} R E l}+3 \dot{\mathrm{R}}\left\{\begin{array}{l}
\ddot{\mathrm{N}} \mathrm{b} \\
\ddot{\mathrm{Ti}}
\end{array}\right. \text { und aus: } \\
& \dot{\mathrm{R} R E} \mathrm{l}+6 \dot{\mathrm{R}}\left\{\begin{array}{l}
\ddot{\mathrm{N} b} \\
\ddot{\mathrm{T} i}
\end{array}\right.
\end{aligned}
$$


5. Fortgesetzte Untersuchungen über die Zusammensetฐung des Aeschynits.

Ich habe bereits folgende Zusammensetzung des Aeschỵits angegeben :

\begin{tabular}{lr} 
Niobsäure & 35,05 \\
Titansäure & 10,56 \\
Zirkonerde (?) & 17,58 \\
Ceroxydul & 15,59 \\
Lanthanerde & 11,13 \\
Yttererde & 4,62 \\
Eisenoxydul & 4,32 \\
Wasser & 1,66 \\
Fluor & Spur \\
\cline { 2 - 2 } & $100,51$.
\end{tabular}

Von der mit Zirkonerde (?) bezeichneten Substanz habe ich bereits bemerkt, dass ich daran zweifeln müsse, dass sie ächte Zirkonerde enthalte; indem sie zwar die allgemeinen Eigenschaften der Zirkonerde besitze, sich aber von der ächten Zirkonerde doch wesentlich durch den Umstand unterscheide, dass ihre Verbindung mit Salzsäure nicht zum Irrystallisiren gebracht werden könne, sondern zu einem gelben Firniss eintrockne, wāhrend die salzsaure Zirkonerde doch so leicht krystallisire, dass sie darin nicht einmal durch einen Gehalt von $50 \frac{\mathrm{o}}{0}$ Titansäure gehindert werde. Auch hatte ich mich bereits davon äberzeugt, dass diese Substanz noch viel Titansäure enthielt; doch war mir damals kein Mittel bekannt, sie davon zu befreien.

Dieses Mittel habe ich gegenwärtig in der Destillation ihres Chlorids gefunden. Dabei ergab es sich, dass diese Substanz in der That gar keine Zirkonerde enthielt, sondern aus einem Gemenge von Titansäure und Ceroxyd bestand. Der Aeschynit enthält also, als wesentlichen Bestandtheil, gar keine Zirkonerde; doch mŏgen Fülle vorkommen, wo auch im Aeschynite andere Basen durch Zirkonerde vertreten werden, wie wir diess beim Pyrochlor gesehen haben.

Der Gang der Analyse, den ich bei dieser neuen Untersuchung befolgte, war folgender.

Das feine Pulver des Aeschynits wurde geglüht und dadurch sein Wassergehalt bestimmt. Der Gewichts-Verlust betrug $1,20 \frac{\mathrm{n}}{0}$. Das geg|ühte Mineral-Pulver wurde mit seiner sechsfachen Menge sauren schwefelsauren Kalis zum glühenden klaren Fluss gebracht. Die erkaltete Salzmasse wurde fein zerrieben und Journ. f. prakt. Chemie L. 3. 
mit warmem, aber nicht kochendem Wasser behandelt. Hierbei blieb schwefelsaure Niobsäure ungelüst, die aber noch nicht rein war. Sie wurde abermals mit saurem schwefelsaurem Kali umgeschmolzen und mit warmem Wasser ausgewaschen. Die schwefelsaure Niobsaure wurde ausgeglüht, wobei $33,20 \%$ reine Sämre zurückblieben. Die vereinigten Flüssigkeiten wurden mit überschïssigem Ammoniak versetzt. Es entstand dadurch ein dicker Niederschlag, der alle anderen Bestandtheile des Aeschynits enthiielt; denn weder hydrothionsaures Ammoniak, noch kleesaures noch phosphorsaures Ammoniak brachten in der von diesem Niederschlage algelaufenen Flüssigkeit dic geringsten Trülungrn hervor.

Der Ammoniak-Niederschlag wurde mit saurem schwefelsanrem Natron geschmolzen und das saure Salz in warmen Wasser gelúst. Die Lúsung wurde so viel wie möglich mit kohlensaurem Ammoniak neutralisirt und hierauf nit einer reichlichen und überschüssigen Henge von kleesaurem Ammoniak ver'setzt. Es entstand dadurch ein Niederschlag, der die grösste Menge des Cers, alles Lanthan und alle Yttererde enthielt, dem aber auch eine nicht unbeträchtliche Henge Titansăure beigemengt war.

Man glühte diesen Niederschlag aus, behandelte die dadurch entstandenen Oxyde mit Schwefelsïure, verjagte die überschüssige Schwefelsiure durch Erhitzen und lüste das Salz in kaltem Wasser. Dahei blieb viel schwefelsaure Titansäure ungelöst. Yan schied sie nicht erst ab, da auch die Lösang noch etwas Titansäure enthielt, sondern sãttigte die Flüssigkeit kochend mit schwefelsaurem Kali. Dadurch wurde der Rest der Titansăure und alles Cer und Lanthan als Doppelsalz niedergeschlagen. Aus der, von diesem Niederschlage abtiltrirten Flüssigkeit, wurden 1,28 Y Yttererde gefällt.

Das Doppelsaiz mit der ihm beigemengten schwefelsauren Titansäure wurle mit lechendem Wasser behandelt. Dadurch lüsten sich die Doppelsalze von Cer und Lanthan auf; die schwefelsaure Titansäure blieb ungelöst. Letztere wurde mit der später erhaltenen Titansãure vereinigt und in Chlorid verwandelt. das Ceroxyd und die Lanthanerde aber durch verdünnte Salpetersäure getrennt. Die von dem Niederschlage durch kleesaures Ammoniak abfiltrirte Flüsigkeit musste jetzt noch den Rest der 
Titansäure, alles Eisenoxyd, etwas Ceroxyd und im Fall der Aeschynit Zirkonerde enthielt, auch die ganze Menge dieser Erde enthalten, da schwefelsaure Zirkonerde durch kleesaures Ammoniak nicht gefällt wird.

Man versetzte diese Flüssigkeit mit Weinsteinsãure und überschüssigem Ammoniak. Hierauf wurde durch hydıothionsaures Ammoniak, das Eisen als Schwefeleisen niedergeschlagen. Dieses gab nach der Umwandlung in Eisenoxyd ein dequivalent

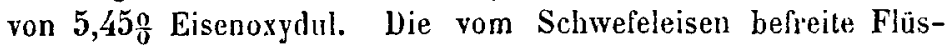
sigkeit wurde eingedampft, die Salzmasse in glïhenden Fluss gebracht und nach Zusatz von üherschüssiger Schwefelsüure abermals geschmolzen. Das saure Salz wurde in Wasser gelüst und die Lüsung durch Ammoniak gefält. Dieser Niederschlag wurde mit der früher erhaltenen schwefelsauren Titansäure vereinigt und stark geglüht. Das hierbei entstandene Oxyd wurde gewogen.

Das abgewogene Oxyd wurde mit der Hälfte seines Gewichts Kohlenpulver und mit einer doppelten Menge Zucker innig gemengt und dieses Gemenge in einem bedeckiten Platintiegel ausgeglüht. Man erhielt dadurch eine poröse kohlige Masse, die in Stücke von der Grösse der Erbsen geschnitten wurde. Diese brachte man in einem Porcellanrohre zum Glühen und leitete trocknes Chlorgas darüber. Hierbei entstanden Chloride von sehr verschiedener Flüchtigkeit. Cerchlorür ist nicht flüchtig und bleibt in der Kohle. Zirkonchlorid ist nur wenig flüchtig und setzt sich gleich hinter der Koble im Porcellanrohr ab. Titanchlorid ist sehr flüchtig und kann nur durch Abbühlung der Vorlagen, die mit dem Porcellanrolure verbunden werden müssen, als eine klare Flüssigkeit dargestellt werden.

Der Apparat, den ich zu diesen Versuchen anwandte, hatte folgende Einrichtung.

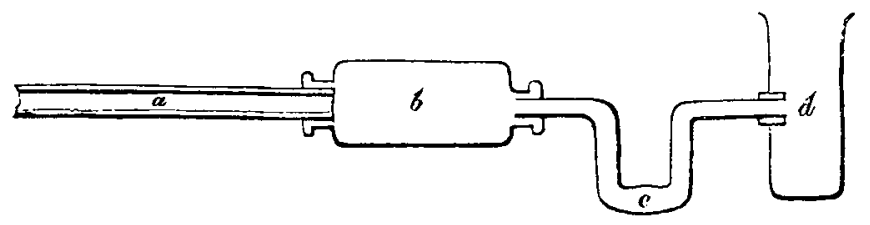

a Porcellanrohr;

6 erster Vorstoss aus Glas; 
c zweiter Vorstoss aus Glas;

d Gefäss mit Kalkhydrat.

Zu Anfang der Operation verdichtete sich etwas Titanchlorid in $b$. Da aber dieser erste Vorstoss nicht abgekühlt wurde, so ging es bald, ausgetrieben durch die heissen Chlordämple in den kalt gehaltenen zweiten Vorstoss $c$ über und verdichtete sich daselbst als eine klare Flüssigkeit.

Das überschüssige Chlor wurde von dem Kalkhydrate des Gefässes $d$ verschluckt.

IIan leitete so lange Chlorgas durch den Apparat, als sich weisse Nebel bildeten, dann liess man ihn erkalten und nahm ilı auseinander.

Der Inhalt des Porcellanrohres wurde jetzt näher untersucht. von einem Sublimate von Zirkonchlorid war in diesem Rohre nichts zu bemerken. Dagegen waren die Wănde des Porcellanrohrs sowohl, als auch die Wände des Vorstosses $b$ mit einer dünnen Schicht eines weissen in Wasser unlóslichen Sublimats überzogen, velches sich bei näherer Untersuchung als Titanacichlorid erwiess.

Das Porcellanrohr wurde nun mit seinem kohligen Inhalte mit Wasser ausgespült; ebenso der Vorstoss $b$. Die Lósung wurde von der Kohle abfiltrirt und letztere ausgewaschen. Man verbrannte nun die Koble und untersuchte, ob sich nicht in der Asche etwas der ursprünglich mit liohle gemengten Oxyde, die sich vielleicht der Einwirkung des Chlors entzogen haben konnten, vorfände, was aber nicht der Fall war.

Die wässrige Lösung wurde mit Ammoniak versetzt. Es entstund dadurch ein Niederschlag, der in Schwefelsäure gelöst wurde. Die neutralisirte Lossung gab mit kleesaurem Ammoniak einen Niederschlag von kleesaurem Ceroxydul. In der von diesem Niederschlag abgelaufenen Flüssigkeit brachte Ammoniak keine Spur eines Niederschlags hervor. Der untersuchte Aeschynit enthielt also keine Spur Zirkonerde.

Das kleesaure Ceroxydul wurde durch Glühen an der Luf in Ceroxyd verwandelt unl gewogen. Sein Gewicht, abgezogen von dem Gewichte der 0xyde, die u'sprunglich der Behandlung mit Chlor unterworfen worden waren, gab das Gewicht der im Aəschỵnit enthaltenen Titansäure, nämlich 25,90․ㅡㅇ. Es bliebe jetzt noch übrig zu untersuchen: in welchem Oxydations- 
zustande befinden sich die in Aeschynit enthaltenen Basen? Ich habe aber diese Frage schon oben, beim Tantalit beantwortet. Danach war im Aeschynit ein Aequivalent von 22,20\% Ceroxyd enthalten.

Als Resultat dieser Analyse des Aeschynits erhielt man:

\begin{tabular}{|c|c|c|c|c|c|}
\hline \multirow{9}{*}{$\begin{array}{l}\text { Niobsäure } \\
\text { Titansäure } \\
\text { Ceroxyd } \\
\text { Ceroxydul } \\
\text { Lanthanerde } \\
\text { Yttererde } \\
\text { Eisenoxydul } \\
\text { Wasser }\end{array}$} & \multicolumn{3}{|c|}{ Sauerstoff. } & \multicolumn{2}{|c|}{$\begin{array}{l}\text { Gefundene Angenommene } \\
\text { Proportion. Proportion. }\end{array}$} \\
\hline & 33,20 & $4,57\}$ & 14,85 & 9,70 & 10 \\
\hline & 25,90 & 10,286 & & 300 & 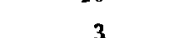 \\
\hline & $\begin{array}{r}22,20 \\
5,12\end{array}$ & $\begin{array}{l}4,59 \\
0,75)\end{array}$ & & & \\
\hline & 6,22 & 0,89 & 310 & 3 04 & $?$ \\
\hline & 1,28 & $0,25\}$ & 3,10 & $2,0=$ & 2 \\
\hline & 5,45 & $1,21)$ & & & \\
\hline & 1,20 & & & & \\
\hline & 100,57 & & & & \\
\hline
\end{tabular}

Hiernach entspricht die Zusammensetzung des Aeschỵnits der Formel :

$$
2\left\{\begin{array}{l}
\dot{\mathrm{C} e} \\
\dot{\mathrm{L} n} \\
\dot{\mathrm{Fe}}
\end{array}\right\} \begin{aligned}
& \ddot{\mathrm{N}} \mathrm{T} \mathrm{T} i \\
& \ddot{\mathrm{T}} \mathrm{E} e
\end{aligned}+\left\{\begin{array}{l}
\ddot{\mathrm{N}} b_{3} \\
\ddot{\mathrm{Ti}_{3}} .
\end{array}\right.
$$

\section{6) Systematisclie Eintheilung der Tantaterze.}

Geht man bei der systematischen Eintheilung der Hineralien von dem chemischen Standpunkte aus und legt man dabei die Eintheilung nach den elektro-negativen Bestandtheilen zu Grunde; so dürften streng genommen in die Familie der Tantalerze nur die eigentlichen Tantalate, also nur Tantalit und Ytterotantalit aufgenommen werden. Da wir aber die wahre Natur der tantalähnlichen Substanzen vieler Mineralien, namentlich des Mikroliths, Fergusonits, Polykrases, Euxenits und Woblerits noch nicht kennen; da es ferner die grosse Schwere der tantalähnlichen Substanzen des bayrischen Columbits wahrscbeinlich macht, dass denselben Tantalsãure beigemengt sei, so lässt sich bis jetzt noch keine strenge Grenzlinie zwischen den Tantalaten, Niobiaten und Ilmeniaten ziehen. Es bleibt daher nichts übrig, als alle diese Körper zu einer Familie der T'antalerze zu vereinigen. Dagegen müssen Mengit und Polymignit, obgleich sie dieselbe. Form wie Columbit haben, aus dieser Familie ausgeschlossen bleiben, weil in diesen Mineralien ein Gehalt von tantalälnnlichen 
Süuren noch nicht mit Bestimmtheit nachgewiesen worden ist. Bis jetzt gehören diese Mineralien in die Familie der Titanerze. Desgleichen bilden die in der Form des Columbits und Fergusonits krystallisirenden Wolframiate und Molybdänate besondere Familien.

Die Tantalerze zerfallen in drei Unterabtheilungen; nämlich:

a) in Verbindungen der tantalähnlichen Säuren mit $\dot{R}$;

b) in Verbindungen derselben mit $\dot{R}$ und $\dddot{R}$;

c) in Verbindungen von Tantalaten mit Silicaten.

Jede dieser Unterabtheilungen enthält Gruppen von MineraJien, die durch gleiche Form charakterisirt werden.

Jede Species besitzt eine besondere stöchiometrische Constitution.

Varietäten werden besonders durch isomorphen Austausch und durch heteromeres Zusammenkrystallisiren, also besonders durch hierdurch bewirkte Abweichungen in den physischen Eigenschaften bellingt. Sie entstehen auch durch Beimengung fremdartiger Körper und durch Pseudomorphose.

Auf diese Principien gründet sich folgende Eintheilung der Tantalerze.

\section{Familie: Tantalerze.}

A. Verbindungen tantalähnlicher Säuren mit $\dot{\mathbf{R}}$.

a) Pyrochlor-Gruppe.

1) Mikrolith $=(\dot{\mathbf{R}} \ddot{\mathrm{T}} \mathrm{a}(?))$ ?.

2) Hydrochlor $=(\dot{\mathrm{R}} \ddot{\mathrm{Ta}}($ ? $)+\dot{\mathrm{H}})$ ?

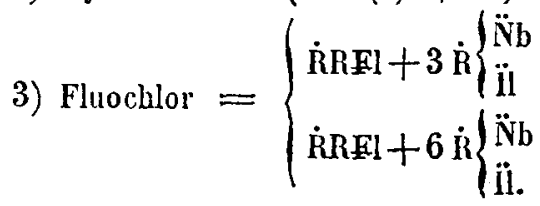

b) Fergusonil-Gruppe.

4) Fergusonit $=\dot{\mathrm{Y}}, \dot{\mathrm{C} e}, \dot{\mathrm{Z}} \mathrm{r}, \ddot{\mathrm{T}} \mathrm{a}(?)$. 
c) Columbit-Gruppe.

5) Columbit.

Varietãten:

$\alpha$. Bayrischer Columbit $=\dot{\mathrm{R}}_{2}\left\{\begin{array}{l}\ddot{\mathrm{p}_{p_{3}}} \\ \ddot{\mathrm{N}} \mathrm{b}_{3}\end{array}\right.$.

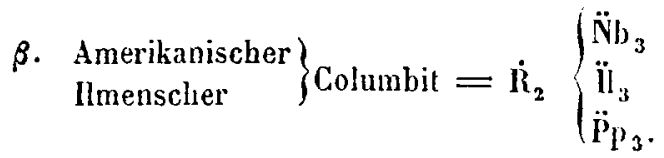

6) Polykras $=\dot{\mathrm{Z}}$, $\dot{\mathrm{Fe}}, \dot{\mathrm{U}}, \dot{\mathrm{C}} \mathrm{e}, \mathrm{Ti}$, $\underset{\mathrm{T}}{\mathrm{T}}(?)$.

7) Ytteroilmenit $=\dot{\mathrm{R}}\left\{\begin{array}{l}\ddot{\mathrm{u}} \\ \ddot{\mathrm{T}} \mathrm{i}\end{array}\right.$

8) Samarskit $=\dot{\mathrm{R}}_{2} \ddot{\mathrm{N}} \mathrm{b}$.

d) Yiterotantalit-Gruppe.

9) Ytterotantalit $=\dot{\mathrm{R}}_{\mathrm{z}} \mathrm{T} \mathrm{i}$.

10) Euxenit $=\dot{\mathrm{V}}, \dot{\mathrm{U}}, \ddot{\mathrm{T}} \mathrm{i}, \ddot{\mathrm{T}} \mathrm{a}(?)$.

B. Verbindungen tantalähnlicher Säuren uit $\dot{k}$ und $\dddot{\mathfrak{H}}$.

e) Aeschynit-Gruppe.

11) Aeschynit $=2 \dot{\mathrm{R}}\left\{\begin{array}{l}\ddot{\mathrm{N}} \mathrm{b} \\ \ddot{\mathrm{T} i}\end{array}+\dddot{\mathrm{R}}\left\{\begin{array}{l}\ddot{N}_{\mathrm{b}_{3}} \\ \dddot{\mathrm{T}}_{3}\end{array}\right.\right.$.

f) Tantalit-Gruppe.

12) Tantalit.

Varietäten :

a. $\quad$ Siderotantal $=\dot{\mathrm{R}}_{2} \ddot{\mathrm{T}} \mathrm{a}_{3}+\dddot{\mathbf{R}} \ddot{\mathrm{T}} \mathrm{a}_{3}$.

Syn. Kimito-Tantalit, Tamela-Tintalit.

$\beta . \quad$ Kassiterotantal $=\left(\dot{\mathrm{R}}_{2} \ddot{\mathrm{T}}_{3}+\dddot{\mathrm{R}} \mathrm{Ta}_{3}\right)+x\left\{\begin{array}{l}\ddot{\mathrm{S} n} \\ \ddot{\mathrm{W}} .\end{array}\right.$

Syı. Finbo-Tantalit, Broddbo-Tantalit. 
C. Verbindungen von Tantalaten mit Silicaten.

13) Wöhlerit $=\dot{\mathrm{Z}} \mathrm{r}, \dot{\mathrm{C} a}, \dot{\mathrm{N}} \mathrm{a}, \ddot{\mathrm{S}} \mathrm{i}, \ddot{\mathrm{T}} \mathrm{a}(?)$.

\section{XXVIII.}

Ueber die Identität von Arkansit und Brookit.

\section{Von \\ R. Hermann $\bullet$ ).}

Kürzlich erhielt ich eimige neue Mineralien von Hot-SpringCounty in Arkansas in Nordamerika, die von Shepard: Arkansit, Ozarkit und Shorlamit benannt worden sind. - Was den Arkansit anbelangt, so muss ich bemerken, dass er identisch ist mit Brookit, obgleich die Form beider Mlineralien auf den ersten Blick ganz verschieden $z u$ sein scheint.

Das Exemplar von Arkansit, welches ich besitze, bebesteht aus einer Druse von Krystallen lichtgrauen Quarzes, die ühersäet ist mit erbsengrossen Krystallen eines schwarzen Minerals. Dieses ist der Arkansit.

Die Iirystalle finden sich meist einzeln, mitunter auch verwacbsen. Ihre Farbe ist schwarz; undurchsichtig, Metallglanz, Härte des Feldspaths, Spec. Gew. 3,79.

Die Krystalle scheinen auf den ersten Blick aus HexagonalDodecaëdern zu bestehen. In der That schwanken die Winkel der Seitenkanten dieses scheinbaren Dodecaëders nur um $\frac{2}{2}$ Grad. Sie betragen nämlicb $100^{\circ} 30^{\prime}-101^{\circ}$. Bei genauerer Untersuchung zeigen aber zwei gegenüberliegende FlächenPaare Spuren von Spaltbarkeit, die an den Andern nicht zu bemerken ist; auch sind diese der Spaltungs-Richtung parallelen Flächen stets glänzender als die andern. Ferner zeigen mehrere Krystalle Spuren von Zuschärfung der Endecken durch zwei auf gegenüberliegende Endkanten aufgesetzte Flächen. Diese Krystalle können daher nicht in das $\mathbf{3 -}$ und $\mathbf{1}$ axige Sỵstem ge-

*) Vergl. Rammelsberg in Pogg. Annal. LXXVII, 586, im Auszuge dessen 4. Supplement zum Handwörterbuche S. $271 . \quad$ D. Red. 